\title{
Plane wave limit of local conserved charges
}

\author{
Andrei Mikhailov \\ California Institute of Technology \\ 452-48, Pasadena CA 91125, U.S.A., and \\ Institute for Theoretical and Experimental Physics \\ 117259, Bol. Cheremushkinskaya, 25, Moscow, Russia \\ E-mail: andrei@theory.caltech.edu
}

ABSTRACT: We study the plane wave limit of the Bäcklund transformations for the classical string in AdS space times a sphere and obtain an explicit expression for the local conserved charges. We show that the Pohlmeyer charges become in the plane wave limit the local integrals of motion of the free massive field. This fixes the coefficients in the expansion of the anomalous dimension as the sum of the Pohlmeyer charges.

Keywords: Penrose limit and pp-wave background, Integrable Equations in Physics, AdS-CFT and dS-CFT Correspondence, Differential and Algebraic Geometry. 


\section{Contents}

1. Introduction 1

2. $A d S_{5} \times S^{5}$ and the plane wave 3

2.1 A special choice of coordinates in $A d S_{5} \times S^{5}$

2.2 An approximate solution for the classical string 1

2.3 Relation between $\mu$ and angular momentum

3. Bäcklund transformations and Pohlmeyer charges 5

3.1 Bäcklund transformations

3.2 Pohlmeyer charges 6

3.3 A relation between $\mathcal{E}_{+}$and $\mathcal{E}_{-}$

4. An action variable in the plane wave limit 8

4.1 Action variable and local conserved charges of the free massive theory

4.2 Integral formula 9

4.3 Expansion in powers of $n / \mu$

4.4 An infinite linear combination of local conserved charges 10

\section{Introduction}

AdS/CFT correspondence is a strong/weak coupling duality. It maps the strongly coupled $\mathcal{N}=4$ Yang-Mills theory to the type-IIB superstring theory on $A d S_{5} \times S^{5}$. It is a useful tool for studying the dynamics of the strongly coupled Yang-Mills theory, but it would be even more important to use this duality to learn more about the string theory. AdS/CFT correspondence relates the string theory to the gauge field theory which at this time is understood much better then the string theory. It would be useful to learn how to rewrite the gauge theory in stringy degrees of freedom.

Recently the classical strings in $A d S_{5} \times S^{5}$ were identified on the gauge theory side as coherent states in the sector of single-trace operators with the large R-charge [1 - 7]. The dynamics of such states is essentially classical because they are composed of the large number of partons (a large number of degrees of freedom).

It was conjectured that the number of partons is in the classical limit a conserved quantity [8]. We have argued in [9] (using the results of [10-12]) that this conserved quantity corresponds to a hidden symmetry of the classical string theory in $A d S_{5} \times S^{5}$. 
Classical string in $A d S_{5} \times S^{5}$ is an integrable system [13-18]. An infinite family of local conserved charges was found by Pohlmeyer [19]. The hidden symmetry corresponding to the length of the operator is generated by an action variable; it is an infinite linear combination of the Pohlmeyer charges.

In this paper we will fix the coefficients of this infinite linear combination by considering the plane wave limit [20]. In the plane wave limit the worldsheet theory becomes the theory of free massive fields. Our action variable in this limit counts the total number of oscillators ${ }^{1}$ in the excited state. We will apply the Bäcklund transformation in the plane wave limit and obtain in this limit an explicit expression for the generating function of the local conserved charges. We will find that the Pohlmeyer charges become the local conserved charges of the free massive fields, and that the oscillator number is indeed an infinite linear combination of them.

The explicit expression for the action variable and the arguments of [22] allow us to answer the following question: given the classical string worldsheet in $A d S_{5} \times S^{5}$, how to compute the anomalous dimension of the corresponding field theory operator? The answer is given by eq. (4.16).

Integrability is a very useful feature of the AdS/CFT correspondence, and the plane wave limit provides an interesting tool for understanding the integrable structures. The worldsheet sigma-model can be understood in some regime as an integrable perturbation of the free massive theory. Integrable structure of the worldsheet theory was studied from this point of view in 23-25]. In particular, the plane wave limit of the commuting conserved charges (and the $1 / R^{2}$ corrections) was studied on the classical and quantum level in [24, 25]. The way we use the plane wave limit here is somewhat similar to considerations in sections 2 and 3 of [26].

Note added in the revised version. In our work we use the Pohlmeyer's definition of the local conserved charges based on the Bäcklund transformations. There is an alternative definition using the spectral invariants of the monodromy matrix, the quasimomenta. There is a particular linear combination of the quasimomenta which in the sector of fast moving strings has a regular expansion in the powers of the spectral parameter $x$ near $x=1$ and near $x=-1$ giving the left and right Pohlmeyer charges. The relation between this definition and the Pohlmeyer's definition was explained in [27]. The expression for the anomalous dimensions in terms of the quasimomenta was obtained in [15-17]. We believe that Bäcklund transformations are important in the theory of integrable systems, and the advantage of the old Pohlmeyer's definition is that it emphasizes the relation between the local conserved charges and the Bäcklund transformations. The relation between the action variable and the Bäcklund transformations is discussed in [28, 29].

The main subject of this paper is the fact that there is an action variable which has a linear expansion in the Pohlmeyer charges. In [28] and [29] we show that this is a consequence of the fact that the Bäcklund transformations are generated by the Pohlmeyer charges.

\footnotetext{
${ }^{1}$ The oscillator number for strings in AdS was previously discussed in 21. I want to thank A. Tseytlin for pointing my attention to this work.
} 
The relation between our formula for the anomalous dimension and the results of [15]17] is discussed in 29].

\section{2. $A d S_{5} \times S^{5}$ and the plane wave}

\subsection{A special choice of coordinates in $A d S_{5} \times S^{5}$}

Let us consider the embeddings of $A d S_{5}$ as the hyperboloid $A d S_{5} \subset \mathbf{R}^{2+4}$ and $S^{5}$ as the sphere $S^{5} \subset \mathbf{R}^{6}$. Let $X_{-1}, X_{0}, \ldots, X_{4}$ denote the coordinates of $\mathbf{R}^{2+4}$ and $Y_{1}, \ldots, Y_{6}$ the coordinates of $\mathbf{R}^{6}$. The hyperboloid and the sphere are given by the equations

$$
X_{-1}^{2}+X_{0}^{2}-\sum_{i=1}^{4} X_{i}^{2}=1, \quad \sum_{i=1}^{6} Y_{i}^{2}=1 .
$$

The metric of $A d S_{5} \times S^{5}$ is:

$$
d s^{2}=R^{2}\left[-d X_{-1}^{2}-d X_{0}^{2}+\sum_{I=1}^{4} d X_{I}^{2}+\sum_{J=1}^{6} d Y_{J}^{2}\right] .
$$

We will consider the string localized near the equator of the sphere. The following parametrization of $A d S_{5} \times S^{5}$ is useful:

$$
\begin{aligned}
X_{-1}+i X_{0} & =\left(1+\epsilon^{2} \sum_{j=1}^{4} x_{j}^{2}\right)^{\frac{1}{2}} \exp \left[i\left(x_{+}-\frac{\epsilon^{2}}{2} x_{-}\right)\right] \\
Y_{5}+i Y_{6} & =\left(1-\epsilon^{2} \sum_{j=1}^{4} y_{j}^{2}\right)^{\frac{1}{2}} \exp \left[i\left(x_{+}+\frac{\epsilon^{2}}{2} x_{-}\right)\right] \\
X_{i} & =\epsilon x_{i}, \quad Y_{i}=\epsilon y_{i}, \quad(i=1,2,3,4)
\end{aligned}
$$

Here $\epsilon$ is a small parameter which is usually chosen to be of the order $1 / R$. We will use the conformal coordinates on the worldsheet. This means that the embedding $x(\tau, \sigma)$ satisfies the constraints:

$$
\begin{aligned}
\left(\partial_{\tau} X\right)^{2}+\left(\partial_{\sigma} X\right)^{2}+\left(\partial_{\tau} Y\right)^{2}+\left(\partial_{\sigma} Y\right)^{2} & =0 \\
\left(\partial_{\tau} X, \partial_{\sigma} X\right)+\left(\partial_{\tau} Y, \partial_{\sigma} Y\right) & =0
\end{aligned}
$$

With these constraints the equations of motion are:

$$
\begin{array}{r}
\left(\partial_{\tau}^{2}-\partial_{\sigma}^{2}\right) X-\left[\left(\partial_{\tau} X, \partial_{\tau} X\right)-\left(\partial_{\sigma} X, \partial_{\sigma} X\right)\right] X=0 \\
\left(\partial_{\tau}^{2}-\partial_{\sigma}^{2}\right) Y+\left[\left(\partial_{\tau} Y, \partial_{\tau} Y\right)-\left(\partial_{\sigma} Y, \partial_{\sigma} Y\right)\right] Y=0
\end{array}
$$

In the plane wave perturbation theory the embedding $x(\tau, \sigma)$ has a regular expansion in powers of $\epsilon$. Let us describe the first approximation. 


\subsection{An approximate solution for the classical string}

Let $\mu$ be a constant parameter. There is an approximate solution for which the eight "transverse" coordinates $x_{1}, \ldots, x_{4}, y_{1}, \ldots, y_{4}$ satisfy the plane wave equations of motion

$$
\begin{aligned}
\left(\partial_{\tau}^{2}-\partial_{\sigma}^{2}\right) x_{i}+\mu^{2} x_{i} & =0 \\
\left(\partial_{\tau}^{2}-\partial_{\sigma}^{2}\right) y_{i}+\mu^{2} y_{i} & =0
\end{aligned}
$$

with the constraint

$$
\int_{0}^{2 \pi} d \sigma\left[\left(\partial_{\tau} x_{i}, \partial_{\sigma} x_{i}\right)+\left(\partial_{\tau} y_{i}, \partial_{\sigma} y_{i}\right)\right]=0
$$

To describe this solution we have to define $x_{+}(\tau, \sigma)$ and $x_{-}(\tau, \sigma)$. Let us introduce the functions $x_{-, A}(\tau, \sigma)$ and $x_{-, S}(\tau, \sigma)$ defined by the equations:

$$
\begin{aligned}
& \partial_{\tau} x_{-, A}=-\frac{1}{2 \mu} \sum_{i=1}^{4}\left[\left(\partial_{\tau} x_{i}\right)^{2}+\left(\partial_{\sigma} x_{i}\right)^{2}-\mu^{2} x_{i}^{2}\right]-\partial_{\tau} \phi \\
& \partial_{\sigma} x_{-, A}=-\frac{1}{\mu} \sum_{i=1}^{4}\left(\partial_{\tau} x_{i}, \partial_{\sigma} x_{i}\right)-\partial_{\sigma} \phi \\
& \partial_{\tau} x_{-, S}=-\frac{1}{2 \mu} \sum_{i=1}^{4}\left[\left(\partial_{\tau} y_{i}\right)^{2}+\left(\partial_{\sigma} y_{i}\right)^{2}-\mu^{2} y_{i}^{2}\right]+\partial_{\tau} \phi \\
& \partial_{\sigma} x_{-, S}=-\frac{1}{\mu} \sum_{i=1}^{4}\left(\partial_{\tau} y_{i}, \partial_{\sigma} y_{i}\right)+\partial_{\sigma} \phi
\end{aligned}
$$

where $\phi$ is an arbitrary function of $\tau, \sigma$ satisfying $\left(\partial_{\tau}^{2}-\partial_{\sigma}^{2}\right) \phi=0$. The results of the calculations in this paper do not depend on the choice of $\phi$. We will use $\phi=c \sigma$ with a constant $^{2} c$. We put:

$$
\begin{aligned}
& x_{+}=\mu\left(\tau-\tau_{0}\right)+\frac{\epsilon^{2}}{2}\left(x_{-, S}-x_{-, A}\right) \\
& x_{-}=\left(x_{-, S}+x_{-, A}\right) .
\end{aligned}
$$

This solves (2.4) approximately, the right hand side of (2.4) is of the order $\epsilon^{3}$. It is possible to modify the definition of $x_{-, A}$ and $x_{-, S}$ by the terms of the order $\epsilon^{2}$ and higher, so that the modified solution solves the constraints (2.3) exactly. These corrections will not spoil the equations of motion (2.4) in the order $\epsilon^{2}$; we will not need the explicit formula for these correcting terms in this paper.

\subsection{Relation between $\mu$ and angular momentum}

The string worldsheet action in the plane wave region is:

$$
\begin{aligned}
S=\frac{1}{2} \frac{1}{2 \pi} \int d \sigma d \tau[ & 2 \partial_{\tau} x_{+} \partial_{\tau} x_{-}-\left(\partial_{\tau} x_{+}\right)^{2}\left(x^{2}+y^{2}\right)+\left(\partial_{\tau} x\right)^{2}+\left(\partial_{\tau} y\right)^{2}+ \\
& \left.+\epsilon^{2} \partial_{\tau} x_{+} \partial_{\tau} x_{-}\left(x^{2}-y^{2}\right)+\epsilon^{2}\left(\left(y, \partial_{\tau} y\right)-\left(x, \partial_{\tau} x\right)^{2}\right)+\cdots\right] .
\end{aligned}
$$

\footnotetext{
${ }^{2}$ A nonzero $c$ may be needed if we want $x_{-, A}$ and $x_{-, S}$ to be periodic functions of $\sigma$. The sum $x_{-, S}(\tau, \sigma)+$ $x_{-, A}(\tau, \sigma)$ is periodic in $\sigma$ because of 2.6).
} 
Here ... denotes the terms which do not contain time derivatives and the terms of the higher order in $\epsilon^{2}$. We will write $y^{2}$ or $(y, y)$ instead of $\sum_{j=1}^{4} y_{j} y_{j}$. The vector field $\partial / \partial x_{-}$is a Killing vector. The corresponding momentum up to the terms of the order $o\left(\epsilon^{2}\right)$ is

$$
\begin{aligned}
M\left(\frac{\partial}{\partial x_{-}}\right) & =\frac{1}{2 \pi} \int d \sigma \partial_{\tau} x_{+}\left(1+\frac{\epsilon^{2}}{2}\left(x^{2}-y^{2}\right)\right) \\
& =\mu-\frac{\epsilon^{2}}{4 \mu} \frac{1}{2 \pi} \int d \sigma\left[\left(\partial_{\tau} y\right)^{2}+\left(\partial_{\sigma} y\right)^{2}+\mu^{2} y^{2}-\left(\partial_{\tau} x\right)^{2}-\left(\partial_{\sigma} x\right)^{2}-\mu^{2} x^{2}\right] .
\end{aligned}
$$

The Killing vector field $\frac{1}{\epsilon^{2}} \frac{\partial}{\partial x_{-}}+\frac{1}{2} \frac{\partial}{\partial x_{+}}$is periodic with the period $2 \pi$. Let us denote $J$ the corresponding angular momentum. We have:

$$
M\left(\frac{\partial}{\partial x_{-}}\right)=\epsilon^{2}\left(J-\frac{1}{2} M\left(\frac{\partial}{\partial x_{+}}\right)\right) .
$$

The momentum corresponding to $\frac{\partial}{\partial x_{+}}$up to the terms of the order $\epsilon^{2}$ is

$$
\begin{aligned}
M\left(\frac{\partial}{\partial x_{+}}\right) & =\frac{1}{2 \pi} \int d \sigma\left[\partial_{\tau} x_{-}-\partial_{\tau} x_{+}\left(x^{2}+y^{2}\right)\right] \\
& =-\frac{1}{2 \mu} \frac{1}{2 \pi} \int d \sigma\left[\left(\partial_{\tau} y\right)^{2}+\left(\partial_{\sigma} y\right)^{2}+\mu^{2} y^{2}+\left(\partial_{\tau} x\right)^{2}+\left(\partial_{\sigma} x\right)^{2}+\mu^{2} x^{2}\right] .
\end{aligned}
$$

Therefore

$$
\mu=\epsilon^{2}\left(J+\frac{1}{2 \mu} \frac{1}{2 \pi} \int d \sigma\left[\left(\partial_{\tau} y\right)^{2}+\left(\partial_{\sigma} y\right)^{2}+\mu^{2} y^{2}\right]\right)+o\left(\epsilon^{2}\right)
$$

\section{Bäcklund transformations and Pohlmeyer charges}

\subsection{Bäcklund transformations}

We will restrict ourselves to the case when $x_{1}=\ldots=x_{4}=0$, which means that the string fluctuates only in $S^{5}$; the projection of the worldsheet to $A d S_{5}$ is a fixed timelike geodesic. We will discuss the general case $x_{i} \neq 0$ at the end of the paper. Consider the Bäcklund transformation which does not change the AdS part of the worldsheet $X$ but changes the $S^{5}$ part $Y \rightarrow Y^{\prime}:$

$$
\begin{gathered}
\partial_{+}\left(Y^{\prime}+Y\right)=\frac{1}{2}\left(1+\gamma^{-2}\right)\left(Y^{\prime}, \partial_{+} Y\right)\left(Y^{\prime}-Y\right) \\
\partial_{-}\left(Y^{\prime}-Y\right)=-\frac{1}{2}\left(1+\gamma^{2}\right)\left(Y^{\prime}, \partial_{-} Y\right)\left(Y^{\prime}+Y\right) .
\end{gathered}
$$

Here $\gamma$ is a constant parameter. We will also use the notation $Y(\gamma)=Y^{\prime}$. Notice that

$$
\left(Y^{\prime}, Y\right)=\frac{1-\gamma^{2}}{1+\gamma^{2}}
$$

Let us apply the transformation $Y \rightarrow Y^{\prime}$ in the plane wave limit. At the zeroth order in $\epsilon^{2}$ we get:

$$
\left(Y^{\prime}, \partial_{+} Y\right)=-\frac{\mu}{2} \sin \left[\mu\left(\tau_{0}^{\prime}-\tau_{0}\right)\right]+O\left(\epsilon^{2}\right)
$$


and $\left(Y^{\prime}, \partial_{-} Y\right)=\left(Y^{\prime}, \partial_{+} Y\right)+O\left(\epsilon^{2}\right)$. Eqs. (3.1) at the zeroth order in $\epsilon^{2}$ :

$$
\begin{aligned}
& \partial_{\tau}\left(e^{i \mu\left(\tau-\tau_{0}^{\prime}\right)}+e^{i \mu\left(\tau-\tau_{0}\right)}\right)=-\frac{\mu}{2}\left(1+\gamma^{-2}\right) \sin \left[\mu\left(\tau_{0}^{\prime}-\tau_{0}\right)\right]\left(e^{i \mu\left(\tau-\tau_{0}^{\prime}\right)}-e^{i \mu\left(\tau-\tau_{0}\right)}\right) \\
& \partial_{\tau}\left(e^{i \mu\left(\tau-\tau_{0}^{\prime}\right)}-e^{i \mu\left(\tau-\tau_{0}\right)}\right)=\frac{\mu}{2}\left(1+\gamma^{2}\right) \sin \left[\mu\left(\tau_{0}^{\prime}-\tau_{0}\right)\right]\left(e^{i \mu\left(\tau-\tau_{0}^{\prime}\right)}+e^{i \mu\left(\tau-\tau_{0}\right)}\right) .
\end{aligned}
$$

This implies that $\tan \left[\frac{\mu}{2}\left(\tau_{0}^{\prime}-\tau_{0}\right)\right]= \pm \gamma$. We will choose the plus sign ${ }^{3}$. Let us introduce $\alpha$ :

$$
\tan \alpha=\gamma
$$

With this notation $\tau_{0}^{\prime}=\tau_{0}+2 \mu^{-1} \alpha$. Then up to the terms of the order $\epsilon^{2}$ we have:

$$
y^{\prime}=\frac{\mu-2 \tan \alpha \partial_{+}}{\mu+2 \tan \alpha \partial_{+}} y=\frac{2 \partial_{-}+\mu \tan \alpha}{2 \partial_{-}-\mu \tan \alpha} y .
$$

We can use (3.2) to find the transformation $x_{-, S} \rightarrow x_{-, S}^{\prime}$ :

$$
x_{-, S}^{\prime}-x_{-, S}=\frac{\cos 2 \alpha}{2 \sin 2 \alpha}\left(y^{2}+\left(y^{\prime}\right)^{2}\right)-\frac{1}{\sin 2 \alpha}\left(y, y^{\prime}\right) .
$$

The parameter $\mu^{-1}$ is the small parameter of the null-surface perturbation theory. In the leading order in $1 / \mu$ we have $\partial_{+} \simeq \frac{1}{2} \partial_{\tau}$ and the Bäcklund transformation is just a time shift:

$$
\begin{aligned}
Y^{\prime} & =\exp \left(-2 \alpha \mu^{-1} \partial_{\tau}\right) Y \\
& =\cos (2 \alpha) Y-\sin (2 \alpha) \mu^{-1} \partial_{\tau} Y \\
& =\frac{1-\gamma^{2}}{1+\gamma^{2}} Y-\frac{2 \gamma}{1+\gamma^{2}} \frac{\partial_{\tau} Y}{\left|\partial_{\tau} Y\right|} .
\end{aligned}
$$

This formula follows directly from (3.1). We have used that $\partial_{\tau}^{2} Y=-\mu^{2} Y$ in the limit $\mu \rightarrow \infty$. Therefore we should consider $\mu^{-1} \partial_{\tau}$ to be of the order 1 in the $1 / \mu$-expansion. We can define the Bäcklund transformations in the null-surface perturbation theory as perturbative solutions of (3.1) which are power series in $\mu^{-1}$, the first approximation given by (3.5). The corrections to (3.5) by the higher powers of $\mu^{-1}$ involve higher derivatives in $\tau$ and $\sigma$ and depend on $\gamma$ as rational functions. When $\gamma$ is small we can expand these corrections in powers of $\gamma$, and when $\gamma$ is large in powers of $\gamma^{-1}$. Therefore the definition of the Bäcklund transformation as a power series in $1 / \mu$ agrees with the usual definition as a power series in $\gamma$ or $1 / \gamma$.

\subsection{Pohlmeyer charges}

The generating function for the conserved charges is given by:

$$
\mathcal{E}(\gamma)=\frac{1}{2 \pi} \int d \sigma\left[\gamma\left(Y(\gamma), \partial_{+} Y\right)+\gamma^{3}\left(Y(\gamma), \partial_{-} Y\right)\right]
$$

\footnotetext{
${ }^{3}$ The relations (3.1) are symmetric in $Y$ and $Y^{\prime}$. Therefore there are two solutions for $Y^{\prime}$. Given one solution $Y^{\prime}=Y(\gamma)$ the other one is $Y(-\gamma)$.
} 


$$
\begin{aligned}
=\gamma \frac{1}{2 \pi} \int d \sigma\{ & -\frac{\sin 2 \alpha}{2 \cos ^{2} \alpha} \mu+ \\
& +\epsilon^{2}\left[\frac{1}{2 \cos ^{2} \alpha}\left(y^{\prime}, \partial_{\tau} y\right)+\frac{1}{2} \frac{\cos 2 \alpha}{\cos ^{2} \alpha}\left(y^{\prime}, \partial_{\sigma} y\right)+\right. \\
& \quad+\frac{1}{2} \frac{\sin 2 \alpha}{\cos ^{2} \alpha}\left[\frac{\mu}{2}\left(y^{2}+\left(y^{\prime}\right)^{2}\right)-\partial_{\tau} x_{-, S}\right]+ \\
& \left.\left.+\frac{1}{2} \frac{\cos 2 \alpha}{\cos ^{2} \alpha}\left[\mu\left(x_{-, S}^{\prime}-x_{-, S}\right)-\left(\partial_{\tau} y, y\right)\right]\right]\right\} .
\end{aligned}
$$

The free field $y$ has an oscillator expansion:

$$
y^{i}(\tau, \sigma)=\sum_{n=-\infty}^{\infty} \sum_{i=1}^{4} \frac{1}{\sqrt{2 \omega_{n}}}\left(\alpha_{n}^{i} e^{i n \sigma+i \omega_{n} \tau}+\overline{\alpha_{n}^{i}} e^{-i n \sigma-i \omega_{n} \tau}\right)
$$

where $\omega_{n}=\sqrt{\mu^{2}+n^{2}}$. We will suppress the index $i$ and write $\sum_{n} \alpha_{n} \overline{\alpha_{n}}$ instead of $\sum_{n=-\infty}^{\infty} \sum_{i=1}^{4} \alpha_{n}^{i} \overline{\alpha_{n}^{i}}$. The Bäcklund transformation for the oscillators is:

$$
y^{\prime}=\frac{\mu-2 \tan \alpha \partial_{+}}{\mu+2 \tan \alpha \partial_{+}} y \quad \Rightarrow \quad \alpha_{n}^{\prime}=\frac{\mu-i \tan \alpha\left(\omega_{n}+n\right)}{\mu+i \tan \alpha\left(\omega_{n}+n\right)} \alpha_{n} .
$$

The oscillator expansion of (3.7) is:

$$
\mathcal{E}(\gamma)=-\gamma^{2} \mu+\epsilon^{2} \frac{\gamma^{2}}{\mu} \mathcal{H}_{2}+\epsilon^{2} \gamma^{2} \hat{\mathcal{E}}_{+}(\gamma)
$$

where

$$
\mathcal{H}_{2}=\frac{1}{4 \pi} \int d \sigma\left[\left(\partial_{\tau} y\right)^{2}+\left(\partial_{\sigma} y\right)^{2}+\mu^{2} y^{2}\right]=\sum \omega_{n} \alpha_{n} \overline{\alpha_{n}}
$$

and

$$
\hat{\mathcal{E}}_{+}(\gamma)=-\sum_{n} \frac{\mu}{\omega_{n}-\cos 2 \alpha n} \alpha_{n} \overline{\alpha_{n}} .
$$

Taking into account (2.13) we get

$$
\mathcal{E}(\gamma)=-\epsilon^{2} \gamma^{2} J+\epsilon^{2} \gamma^{2} \hat{\mathcal{E}}_{+}(\gamma)
$$

This is the generating function for the "left" Pohlmeyer charges. In the generating function for the "right" charges we replace $\hat{\mathcal{E}}_{+}(\gamma)$ with $\hat{\mathcal{E}}_{-}(\gamma)$ which is obtained from $\hat{\mathcal{E}}_{+}(\gamma)$ by replacing $n \rightarrow-n$. We can also consider the average of the left and right generating functions:

$$
\mathcal{E}^{\text {even }}(\gamma)=-\epsilon^{2} \gamma^{2} J+\epsilon^{2} \gamma^{2} \hat{\mathcal{E}}^{\text {even }}(\gamma)
$$

where

$$
\hat{\mathcal{E}}^{\text {even }}(\gamma)=-\sum_{n} \frac{\mu \omega_{n}}{\omega_{n}^{2}-(\cos 2 \alpha)^{2} n^{2}} \alpha_{n} \overline{\alpha_{n}}
$$




\subsection{A relation between $\mathcal{E}_{+}$and $\mathcal{E}_{-}$}

Eq. 3.12 can be also written in the following way:

$$
\begin{aligned}
\hat{\mathcal{E}}_{ \pm}(\gamma) & =-\left(1+\gamma^{2}\right) \sum_{n} \frac{\mu(\omega \pm n)}{\mu^{2}+\gamma^{2}(\omega \pm n)^{2}} \alpha_{n} \overline{\alpha_{n}} \\
& =-\sum_{n} \frac{\mu(\omega \pm n)}{\mu^{2}+\sin ^{2} \alpha\left[(\omega \pm n)^{2}-\mu^{2}\right]} \alpha_{n} \overline{\alpha_{n}} .
\end{aligned}
$$

It is usually understood that $\mathcal{E}(\gamma)$ is the generating function of the conserved charges; it is defined as a power series in $\gamma$. But we see from (3.16) that the expansion in powers of $\sin ^{2} \alpha=\frac{\gamma^{2}}{1+\gamma^{2}}$ is in fact the expansion in powers of the expression $\left[\frac{(\omega \pm n)^{2}}{\mu^{2}}-1\right]$ which is small in the null-surface limit $\mu \rightarrow \infty$. Therefore we can analytically continue $\mathcal{E}(\gamma)$ to finite values of $\gamma$. It follows from $\omega^{2}-n^{2}=\mu^{2}$ that

$$
\hat{\mathcal{E}}_{+}(\gamma)=\hat{\mathcal{E}}_{-}\left(\gamma^{-1}\right) .
$$

This identity follows from the definitions (3.1), (3.6). Eq. (3.1) defines the left Bäcklund transformation $Y \rightarrow Y^{\prime}$. The definition of the right Bäcklund transformation $Y \rightarrow Y^{\prime \prime}$ differs from (3.1) by $\partial_{+} \leftrightarrow \partial_{-}$. With this definition $Y^{\prime}(\gamma)=-Y^{\prime \prime}\left(-\gamma^{-1}\right)$. Now (3.17) follows from the formula (3.6) for the generating function.

\section{An action variable in the plane wave limit}

\subsection{Action variable and local conserved charges of the free massive theory}

The hamiltonian of the plane wave theory is:

$$
H=\frac{1}{4 \pi} \int d \sigma \sum_{i=1}^{4}\left[p_{i}^{2}+q_{i}^{2}+\left(\partial_{\sigma} x_{i}\right)^{2}+\left(\partial_{\sigma} y_{i}\right)^{2}+\mu^{2}\left(x_{i}^{2}+y_{i}^{2}\right)\right]
$$

where $p_{i}(\sigma)=\partial_{\tau} x_{i}$ and $q_{i}(\sigma)=\partial_{\tau} y_{i}$ are the momenta conjugate to $x_{i}$ and $y_{i}$ respectively:

$$
\left\{p_{i}(\sigma), x_{j}\left(\sigma^{\prime}\right)\right\}=2 \pi \delta\left(\sigma-\sigma^{\prime}\right), \quad\left\{q_{i}(\sigma), y_{j}\left(\sigma^{\prime}\right)\right\}=2 \pi \delta\left(\sigma-\sigma^{\prime}\right) .
$$

Consider the following action variable:

$$
\mathcal{I}=\frac{1}{4 \pi} \int d \sigma\left[y_{i}(\sigma) \sqrt{\mu^{2}-\partial_{\sigma}^{2}} y_{i}(\sigma)+q_{i}(\sigma) \frac{1}{\sqrt{\mu^{2}-\partial_{\sigma}^{2}}} q_{i}(\sigma)\right] .
$$

This quantity is conserved and generates periodic trajectories. In the oscillator language:

$$
\mathcal{I}=\sum_{n} \alpha_{n} \overline{\alpha_{n}}
$$

On the other hand the free massive theory has an infinite family of local conserved charges:

$$
\begin{aligned}
I_{k} & =\frac{1}{4 \pi} \sum_{i=1}^{4} \int d \sigma\left[q_{i} \partial_{\sigma}^{2 k-2} q_{i}-y_{i} \partial_{\sigma}^{2 k} y_{i}+\mu^{2} y_{i} \partial_{\sigma}^{2 k-2} y_{i}\right] \\
& =(-1)^{k+1} \times \sum_{n} n^{2 k-2} \omega_{n} \alpha_{n} \overline{\alpha_{n}} .
\end{aligned}
$$


One can see that $\mathcal{I}$ is an infinite linear combination of $I_{k}$ :

$$
\begin{aligned}
\mathcal{I}=\frac{1}{4 \pi} \sum_{i=1}^{4} \int d \sigma[ & \mu y_{i} y_{i}-\sum_{n=0}^{\infty} \mu^{-2 n-1} \frac{(2 n) !}{2^{2 n+1}(n+1) ! n !} y_{i} \partial_{\sigma}^{2 n+2} y_{i}+ \\
& \left.+\sum_{n=0}^{\infty} \mu^{-2 n-1} \frac{(2 n) !}{2^{2 n}(n !)^{2}} q_{i} \partial_{\sigma}^{2 n} q_{i}\right] \\
= & \sum_{n=0}^{\infty} \mu^{-2 n-1} \frac{(2 n) !}{2^{2 n}(n !)^{2}} I_{n+1} .
\end{aligned}
$$

In this section we will show that $I_{k}$ descend from the Pohlmeyer charges of the sigma model on $S^{5}$. We will argue that eq. (4.6) provides an expression for the action variable in terms of the local conserved charges (which is valid not only in the plane wave limit).

\subsection{Integral formula}

There is an integral formula:

$$
\frac{1}{\pi} \int_{-\infty}^{+\infty} \frac{d \gamma}{1+\gamma^{2}} \hat{\mathcal{E}}_{+}(\gamma)=\frac{1}{\pi} \int_{-\infty}^{+\infty} \frac{d \gamma}{1+\gamma^{2}} \hat{\mathcal{E}}_{-}(\gamma)=-\sum_{n} \alpha_{n} \overline{\alpha_{n}}
$$

This means that:

$$
J+\sum_{n} \alpha_{n} \overline{\alpha_{n}}=-\frac{1}{\pi} \int_{-\infty}^{+\infty} \frac{d \gamma}{\gamma^{2}\left(1+\gamma^{2}\right)} \mathcal{E}(\gamma)
$$

This is an integral representation for the action variable in terms of the conserved charges. We derived this formula in the plane wave limit, but we conjecture that it is valid also outside of the plane wave limit. In other words, the right hand side of (4.8) should generate periodic trajectories on the phase space of the classical string in $A d S_{5} \times S^{5}$. We will give an argument in favor of this at the end of section 4.4 .

\subsection{Expansion in powers of $n / \mu$}

Let us rewrite (3.15) in the following way:

$$
\hat{\mathcal{E}}^{\mathrm{even}}(\gamma)=-\sum_{n} \frac{\sqrt{1+(n / \mu)^{2}}}{1+(n / \mu)^{2}(\sin 2 \alpha)^{2}} \alpha_{n} \overline{\alpha_{n}} .
$$

Notice that $(\sin 2 \alpha)^{2}=\frac{4 \gamma^{2}}{\left(1+\gamma^{2}\right)^{2}}$. Let us define the local conserved charges $\hat{\mathcal{E}}_{2 k}$ as follows:

$$
\hat{\mathcal{E}}^{\text {even }}(\gamma)=\sum_{k=0}^{\infty} \gamma^{2 k} \hat{\mathcal{E}}_{2 k}
$$

One can verify the following identities:

$$
\begin{array}{r}
\hat{\mathcal{E}}_{0}+\frac{1}{8} \hat{\mathcal{E}}_{2}=-\sum\left(1+o\left(\frac{n^{2}}{\mu^{2}}\right)\right) \alpha_{n} \overline{\alpha_{n}} \\
\hat{\mathcal{E}}_{0}+\frac{11}{64} \hat{\mathcal{E}}_{2}+\frac{3}{128} \hat{\mathcal{E}}_{4}=-\sum\left(1+o\left(\frac{n^{4}}{\mu^{4}}\right)\right) \alpha_{n} \overline{\alpha_{n}}
\end{array}
$$




$$
\begin{array}{r}
\hat{\mathcal{E}}_{0}+\frac{201}{1024} \hat{\mathcal{E}}_{2}+\frac{11}{256} \hat{\mathcal{E}}_{4}++\frac{5}{1024} \hat{\mathcal{E}}_{6}=-\sum\left(1+o\left(\frac{n^{6}}{\mu^{6}}\right)\right) \alpha_{n} \overline{\alpha_{n}} \\
\hat{\mathcal{E}}_{0}+\frac{3461}{16384} \hat{\mathcal{E}}_{2}+\frac{949}{16384} \hat{\mathcal{E}}_{4}+\frac{185}{16384} \hat{\mathcal{E}}_{6}+\frac{35}{32768} \hat{\mathcal{E}}_{8}=-\sum\left(1+o\left(\frac{n^{8}}{\mu^{8}}\right)\right) \alpha_{n} \overline{\alpha_{n}} .
\end{array}
$$

These equations agree with the results of [10, 11] (see the discussion in [9]). In the next order we have:

$\hat{\mathcal{E}}_{0}+\frac{29011}{131072} \hat{\mathcal{E}}_{2}+\frac{569}{8192} \hat{\mathcal{E}}_{4}+\frac{4661}{262144} \hat{\mathcal{E}}_{6}+\frac{49}{16384} \hat{\mathcal{E}}_{8}+\frac{63}{262144} \hat{\mathcal{E}}_{10}=-\sum\left(1+o\left(\frac{n^{10}}{\mu^{10}}\right)\right) \alpha_{n} \overline{\alpha_{n}}$.

\subsection{An infinite linear combination of local conserved charges}

Instead of expanding $\mathcal{E}$ in powers of $\gamma$ let us now expand it in powers of $(\sin 2 \alpha)^{2}=\frac{4 \gamma^{2}}{\left(1+\gamma^{2}\right)^{2}}$. Define $\mathcal{G}_{k}$ as follows:

$$
\mathcal{E}^{\text {even }}(\gamma)=\epsilon^{2} \gamma^{2} \sum_{k=0}^{\infty}\left[\frac{4 \gamma^{2}}{\left(1+\gamma^{2}\right)^{2}}\right]^{k} \mathcal{G}_{k} .
$$

Then eqs. (4.5) and (4.9) imply that in the plane wave limit

$$
-\mathcal{G}_{k}=\mu^{-1-2 k} I_{k+1}+\delta_{k, 0} J .
$$

Therefore $\mathcal{G}_{k}$ are the "improved" currents of [10, 11]. Indeed, the density of the $k$-th improved current is proportional to $\left|p_{S}\right|^{1-2 k}$ where $p_{S}=\partial_{\tau} x_{S}$ is the push-forward of $\frac{\partial}{\partial \tau}$ by the embedding of the string worldsheet in the $S^{5}$; it is assumed that $\tau$ and $\sigma$ are conformal coordinates on the worldsheet and $\sigma$ is periodic with the period $2 \pi$. The plane wave limit keeps the terms quadratic in $y$. The typical term is $\int d \sigma\left|p_{S}(\sigma)\right|^{1-2 k}\left(\partial_{\sigma}^{k} Y, \partial_{\sigma}^{k} Y\right)$. In the plane wave limit $\left|p_{S}\right| \simeq \mu$ and $Y \simeq \epsilon y$. Therefore in the plane wave limit this quadratic term will be of the form $\epsilon^{2} \mu^{1-2 k} \int d \sigma\left(\partial_{\sigma}^{k} y, \partial_{\sigma}^{k} y\right)$ which is in agreement with (4.13) and (4.5).

We conjecture that the conserved charges $\mathcal{G}_{k}$ defined from the generating function by eq. (4.12) are "exactly improved" (proportional to $|p|^{1-2 k}$ not only in the plane wave limit). We have not proven this. What has to be proven is the existence of the improved charges. Once the existence is proven, the improved charges provide a canonical basis in the space of all the local conserved charges and the coefficients of the expansion (4.12) are just the coefficients of the expansion of $\mathcal{E}$ in this basis. There must be many ways to determine these coefficients, in particular from the plane wave limit, as we did here.

Eqs. (3.13) and (4.9) imply that the action variable is an infinite sum of $\mathcal{G}_{k}$ :

$$
J+\sum_{n=-\infty}^{\infty} \sum_{i=1}^{4} \alpha_{n}^{i} \overline{\alpha_{n}^{i}}=-\sum_{k=0}^{\infty} \frac{1}{2^{2 k}} \frac{(2 k) !}{(k !)^{2}} \mathcal{G}_{k} .
$$

We have so far considered only the $S^{5}$ part of the string worldsheet. Therefore the Pohlmeyer charges $\mathcal{G}_{k}$ which we considered should be really denoted $\mathcal{G}_{k}^{S}$. The index $S$ tells us that these charges came from the $S^{5}$ sigma model. One can introduce in the same way the Pohlmeyer charges $\mathcal{G}_{k}^{A}$ in the $A d S_{5}$ sigma model. We would get:

$$
E-\sum_{n=-\infty}^{\infty} \sum_{i=1}^{4} \beta_{n}^{i} \overline{\beta_{n}^{i}}=-\sum_{k=0}^{\infty} \frac{1}{2^{2 k}} \frac{(2 k) !}{(k !)^{2}} \mathcal{G}_{k}^{A} .
$$


Here $\beta_{n}^{i}$ are the Fourier modes of $x^{i}(\tau, \sigma)$. Arguments of [22] show that the anomalous dimension has the following expansion in these charges:

$$
\frac{1}{2 \pi} \log c^{2}=\sum_{k=0}^{\infty} \frac{1}{2^{2 k}} \frac{(2 k) !}{(k !)^{2}}\left(\mathcal{G}_{k}^{S}-\mathcal{G}_{k}^{A}\right) .
$$

We derived this expression from the plane wave limit, but it should be valid for all classical strings in $A d S_{5} \times S^{5}$, at least in the perturbative expansion around the null-surfaces 9 , 12, 22]. Indeed, we have argued in [9] that there is a universal (in the fast moving sector) expansion of the action variable as an infinite linear combination of the local conserved charges. The coefficients of this expansion are just numbers, and therefore can be fixed from the plane wave limit.

Under the assumption that the charges $\mathcal{G}_{k}$ defined by eq. (4.12) are exactly improved, the integral formula (4.8) is equivalent to the expansion (4.14). Indeed, if we substitute in the integral formula (4.8) the expansion (4.12), we get eq. (4.14).

Eq. (4.16) agrees with the usual definition of the anomalous dimension in the plane wave limit as $E-J-\sum_{n=-\infty}^{\infty} \sum_{i=1}^{4}\left(\alpha_{n}^{i} \overline{\alpha_{n}^{i}}+\beta_{n}^{i} \overline{\beta_{n}^{i}}\right)$.

\section{Acknowledgments}

I want to thank I. Swanson and A. Tseytlin for interesting discussions, and M. Srednicki for asking the question. This research was supported by the Sherman Fairchild Fellowship and in part by the RFBR Grant No. 03-02-17373 and in part by the Russian Grant for the support of the scientific schools NSh-1999.2003.2.

\section{References}

[1] S. Frolov and A.A. Tseytlin, Semiclassical quantization of rotating superstring in $A d S_{5} \times S^{5}$, JHEP 06 (2002) 007 hep-th/0204226.

[2] A.A. Tseytlin, Semiclassical quantization of superstrings: $A d S_{5} \times S^{5}$ and beyond, Int. $J$. Mod. Phys. A 18 (2003) 981 hep-th/0209116.

[3] J.G. Russo, Anomalous dimensions in gauge theories from rotating strings in $A d S_{5} \times S^{5}$, JHEP 06 (2002) 038 hep-th/0205244.

[4] J.A. Minahan and K. Zarembo, The Bethe-Ansatz for $N=4$ super Yang-Mills, JHEP 03 (2003) 013 hep-th/0212208.

[5] S. Frolov and A.A. Tseytlin, Multi-spin string solutions in $A d S_{5} \times S^{5}$, Nucl. Phys. B 668 (2003) 77 hep-th/0304255.

[6] S. Frolov and A.A. Tseytlin, Quantizing three-spin string solution in $A d S_{5} \times S^{5}$, JHEP 07 (2003) 016 hep-th/0306130.

[7] M. Kruczenski, Spin chains and string theory, Phys. Rev. Lett. 93 (2004) 161602 hep-th/0311203.

[8] J.A. Minahan, Higher loops beyond the SU(2) sector, JHEP 10 (2004) 053 hep-th/0405243. 
[9] A. Mikhailov, Notes on fast moving strings, hep-th/0409040.

[10] G. Arutyunov and M. Staudacher, Matching higher conserved charges for strings and spins, JHEP 03 (2004) 004 hep-th/0310182.

[11] J. Engquist, Higher conserved charges and integrability for spinning strings in $A d S_{5} \times S^{5}$, JHEP 04 (2004) 002 hep-th/0402092.

[12] M. Kruczenski and A.A. Tseytlin, Semiclassical relativistic strings in $s^{* *} 5$ and long coherent operators in $N=4$ SYM theory, JHEP 09 (2004) 038 hep-th/0406189.

[13] G. Mandal, N.V. Suryanarayana and S.R. Wadia, Aspects of semiclassical strings in $A d S_{5}$, Phys. Lett. B 543 (2002) 81 hep-th/0206103.

[14] I. Bena, J. Polchinski and R. Roiban, Hidden symmetries of the $A d S_{5} \times S^{5}$ superstring, Phys. Rev. D 69 (2004) 046002 hep-th/0305116.

[15] V.A. Kazakov, A. Marshakov, J.A. Minahan and K. Zarembo, Classical/quantum integrability in AdS/CFT, JHEP 05 (2004) 024 hep-th/0402207.

[16] V.A. Kazakov and K. Zarembo, Classical/quantum integrability in non-compact sector of AdS/CFT, JHEP 10 (2004) 060 hep-th/0410105.

[17] N. Beisert, V.A. Kazakov and K. Sakai, Algebraic curve for the SO(6) sector of AdS/CFT, hep-th/0410253.

[18] S. Schafer-Nameki, The algebraic curve of 1-loop planar $N=4 S Y M$, Nucl. Phys. B 714 (2005) 3 hep-th/0412254.

[19] K. Pohlmeyer, Integrable hamiltonian systems and interactions through quadratic constraints, Commun. Math. Phys. 46 (1976) 207.

[20] D. Berenstein, J.M. Maldacena and H. Nastase, Strings in flat space and pp waves from $N=4$ super Yang-Mills, JHEP 04 (2002) 013 hep-th/0202021.

[21] H.J. de Vega, A.L. Larsen and N. Sanchez, Semiclassical quantization of circular strings in de Sitter and anti-de Sitter space-times, Phys. Rev. D 51 (1995) 6917 hep-th/9410219].

[22] A. Mikhailov, Anomalous dimension and local charges, hep-th/0411178.

[23] L.F. Alday, Non-local charges on $A d S_{5} \times S^{5}$ and pp-waves, JHEP 12 (2003) 033 hep-th/0310146.

[24] I.J. Swanson, On the integrability of string theory in $A d S_{5} \times S^{5}$, hep-th/0405172.

[25] I.J. Swanson, Quantum string integrability and AdS/CFT, Nucl. Phys. B 709 (2005) 443 hep-th/0410282.

[26] M. Kruczenski, A.V. Ryzhov and A.A. Tseytlin, Large spin limit of $A d S_{5} \times S^{5}$ string theory and low energy expansion of ferromagnetic spin chains, Nucl. Phys. B 692 (2004) 3 hep-th/0403120.

[27] G. Arutyunov and M. Zamaklar, Linking baecklund and monodromy charges for strings on $A d S_{5} \times S^{5}$, JHEP 07 (2005) 026 hep-th/0504144.

[28] A. Mikhailov, An action variable of the Sine-Gordon model, hep-th/0504035.

[29] A. Mikhailov, Baecklund transformations, energy shift and the plane wave limit, hep-th/0507261. 